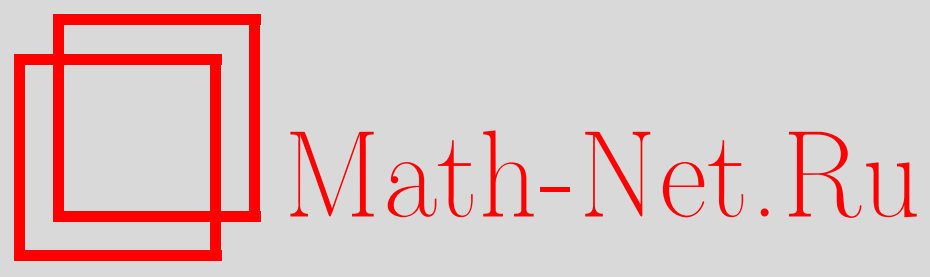

В. П. Курдюмов, А. П. Хромов, О базисах Рисса из собственных функций интегрального оператора с переменным пределом интегрирования, Матем. заметки, 2004, том 76, выпуск 1, 97-110

DOI: https://doi.org/10.4213/mzm92

Использование Общероссийского математического портала Math-Net.Ru подразумевает, что вы прочитали и согласны с пользовательским соглашением http://www . mathnet.ru/rus/agreement

Параметры загрузки:

IP : 35.173 .219 .12

26 апреля 2023 г., 15:55:32

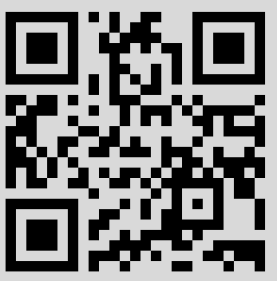




\section{О БАЗИСАХ РИССА ИЗ СОБСТВЕННЫХ ФУНКЦИЙ ИНТЕГРАЛЬНОГО ОПЕРАТОРА С ПЕРЕМЕННЫМ ПРЕДЕЛОМ ИНТЕГРИРОВАНИЯ}

\section{В.П. Курдюмов, А.П. Хромов}

Доказывается теорема о базисности Рисса в пространстве $L_{2}[0,1]$ собственных и присоединенных функций интегрального оператора, некоторая производная ядра которого терпит разрьв на линии $t=1-x$.

Библиография: 20 названий.

В настоящей работе исследуется вопрос о базисности Рисса в пространстве $L_{2}[0,1]$ собственных и присоединенных функций (СП $\Phi)$ интегрального оператора

$$
A f(x)=\int_{0}^{1-x} A(1-x, t) f(t) d t, \quad x \in[0,1] .
$$

Предполагаем, что при $0 \leqslant t \leqslant x \leqslant 1$ существуют и непрерывны производные

$$
\begin{aligned}
& \frac{\partial^{j+s}}{\partial x^{j} \partial t^{s}} A(x, t), \quad j=0,1, \ldots, n, \quad s=0,1, \\
& \left.\frac{\partial^{j}}{\partial x^{j}} A(x, t)\right|_{t=x}=\delta_{n-1, j}, \quad j=0, \ldots, n,
\end{aligned}
$$

где $\delta_{i, j}$ - символ Кронекера.

Операторы $(1),(2)$ и более общего вида, когда ядро $A(x, t)$ допускает разрывы некоторых производных на линиях $t=x$ и $t=1-x$, впервые рассматривались одним из авторов [1]. Была исследована задача точного обращения таких операторов и установлена равносходимость разложений по СП $\Phi$ и в тригонометрический ряд Фурье [2], [3].

Для дифференциальных операторов с усиленно регулярными краевыми условиями базисность Рисса СПФ установлена в [4]-[6]. Эти работы стимулировали большоеколичество исследований. Отметим близкиек нам работы А. А. Шкаликова [7]-[9], А. М. Гомилко и Г. В. Радзиевского [10], [11], А. Г. Баскакова и Т. К. Кацарана [12] по базисам Рисса из СПФ дифференциальных и интегро-дифференциальных операторов с интегральными краевыми условиями. Широкое развитие получил новьй метод исследования базисных свойств СПФ дифференциальных операторов, не привязанных к граничным

Работа выполнена при финансовой поддержке программы Президента РФ "Ведущие научные школы”, грант № НШ-1295.2003.1, и Российского фонда фундаментальных исследований, грант № 00-01-00075. 
условиям, предложенный впервые В. А. Ильиным [13]-[15]. Базисность Рисса СП тегральных операторов свертки установлена Б. В. Пальцевым [16]. Для интегральных операторов с ядрами, имеющими разрьв $(n-1)$-й производной по $x$ на линии $t=x$, установлена в [17], а для оператора (1), (2) при $n=1$ в [18]. Случай $n>1$ значительно более трудный и применяемьй теперь метод является дальнейшим существенным развитием метода из [18].

1. Сведение к интегро-дифференциальному оператору в пространстве вектор-функций. Для определенности будем считать, что $n=4 q$.

Теорема 1. Oператор $A^{-1}$ существует, причем $A^{-1}$ представляет собой интегро-дифференииальный оператор

$$
A^{-1} y(x)=y^{(n)}(1-x)+\int_{0}^{x} N_{1, t}(x, t) y^{(n-1)}(1-t) d t
$$

с краевыми условиями

$$
y^{(k-1)}(1)=0, \quad k=1, \ldots, n
$$

Здесь $N_{1}(x, t)$ - ядро интегрального оператора $N_{1}=\left(E+A_{x^{n}}\right)^{-1}-E, E-$ единичный оператор,

$$
\begin{array}{cc}
A_{x^{n}} f(x)=\int_{0}^{x} A_{x^{n}}^{(n)}(x, t) f(t) d t, & A_{x^{n}}^{(n)}(x, t)=\frac{\partial^{n}}{\partial x^{n}} A(x, t), \\
N_{1, t}(x, \xi)=\left.\frac{\partial N_{1}(x, t)}{\partial t}\right|_{t=\xi}, & y^{\prime}(1-x)=\left.y_{\xi}^{\prime}(\xi)\right|_{\xi=1-x}
\end{array}
$$

ДоКАЗАТЕЛЬСТВо. Положим $y(x)=A f(x)$. Тогда $y(x)$ удовлетворяет $(4)$ и

$$
y^{(n)}(1-x)=f(x)+A_{x^{n}} f(x) .
$$

Отсюда получаем (3).

Обратно, пусть $y(x)$ удовлетворяет (4), и правую часть (3) обозначим через $f(x)$. Проводя здесь интегрирование по частям с учетом $N_{1}(x, x) \equiv 0$, приходим к

$$
y^{(n)}(1-x)=f(x)+A_{x^{n}} f(x)=\frac{d^{n}}{d x^{n}} \int_{0}^{x} A(x, t) f(t) d t .
$$

Отсюда в силу (4) получаем

$$
y(1-x)=\int_{0}^{x} A(x, t) f(t) d t
$$

Теорема доказана.

В пространстве вектор-функций $f(x)=\left(f_{1}(x), f_{2}(x)\right)^{T}(T$ - знак транспонирования) введем интегральньй оператор

$$
N f(x)=\int_{0}^{1 / 2} N(x, t) f(t) d t, \quad x \in\left[0, \frac{1}{2}\right],
$$


где

$$
\begin{gathered}
N(x, t)=B \Gamma^{-1} \tilde{N}(x, t) \Gamma, \quad \tilde{N}(x, t)=\left(\tilde{N}_{i j}(x, t)\right)_{i, j=1}^{2}, \\
\tilde{N}_{11}(x, t)=N_{1, t}\left(\frac{1}{2}+x, \frac{1}{2}-t\right) \varepsilon(t, x), \quad \widetilde{N}_{12}(x, t)=0 \\
\widetilde{N}_{21}(x, t)=N_{1, t}\left(\frac{1}{2}+x, \frac{1}{2}-t\right), \quad \tilde{N}_{22}(x, t)=N_{1, t}\left(\frac{1}{2}+x, \frac{1}{2}+t\right) \varepsilon(x, t),
\end{gathered}
$$

$\varepsilon(x, t)=1$ при $t \leqslant x, \varepsilon(x, t)=0$ при $t>x, B=\left(\begin{array}{cc}1 & 0 \\ 0 & -1\end{array}\right), \Gamma=\left(\begin{array}{cc}1 & 1 \\ 1 & -1\end{array}\right)$.

Обозначим, далее, через $L$ интегро-дифференциальный оператор

$$
L y(x)=B y^{(n)}(x)+N y^{(n-1)}(x), \quad x \in\left[0, \frac{1}{2}\right]
$$

с граничньми условиями

$$
\begin{aligned}
& U_{k}(y)=P_{1} y^{(k-1)}(0)+Q y^{(k-1)}\left(\frac{1}{2}\right)=0, \quad k=2 r-1, \quad r=1, \ldots, \frac{n}{2}, \\
& U_{k}(y)=P_{2} y^{(k-1)}(0)+Q y^{(k-1)}\left(\frac{1}{2}\right)=0, \quad k=2 r, \quad r=1, \ldots, \frac{n}{2},
\end{aligned}
$$

где $P_{1}=\left(\begin{array}{ll}0 & 1 \\ 0 & 0\end{array}\right), P_{2}=\left(\begin{array}{ll}1 & 0 \\ 0 & 0\end{array}\right), Q=\left(\begin{array}{cc}0 & 0 \\ 1 & -1\end{array}\right)$.

ТЕОрема 2. Если $y(x)$ является собственной (присоединенной) функиией оператора $A$ для характеристического числа $\lambda$, то $u(x)=\Gamma^{-1}(y(1 / 2+x), y(1 / 2-x))^{T}$, $x \in[0,1 / 2]$, является собственной (присоединенной) функиией оператора $L$ для собственного значения $\lambda$; и наоборот, если $и(x)$ является собственной (присоединенной) функиией оператора $L$, то $y(x)=(\Gamma u(1 / 2-x)))_{2}$ при $x \in[0,1 / 2]$, $y(x)=(\Gamma u(x-1 / 2))_{1}$ при $x \in[1 / 2,1]$, является собственной (присоединенной) функиией оператора $A$.

ДоКАЗАТЕЛЬСТВО проведем лишь для случая собственных функций (для присоединенных функций оно аналогично, лишь несколько более громоздкое). Пусть $y(x)=$ $\lambda A y(x)$ и $y(x) \not \equiv 0$. Тогда по теореме 1

$$
y^{(n)}(1-x)+\int_{0}^{x} N_{1, t}(x, t) y^{(n-1)}(1-t) d t=\lambda y(x) .
$$

Перейдем в (6) от $x$ к $x+1 / 2$. Тогда при $x \in[-1 / 2,1 / 2]$ получим

$$
y^{(n)}\left(\frac{1}{2}-x\right)+\int_{1 / 2}^{x} N_{1, t}\left(\frac{1}{2}+x, \frac{1}{2}+t\right) y^{(n-1)}\left(\frac{1}{2}-t\right) d t=\lambda y\left(\frac{1}{2}+x\right) .
$$

Положим $z(x)=\left(z_{1}(x), z_{2}(x)\right)^{T}$, где $z_{1}(x)=y(1 / 2+x), z_{2}(x)=y(1 / 2-x), x \in[0,1 / 2]$. Тогда, записывая (7) отдельно при $x \in[0,1 / 2]$ и $x \in[-1 / 2,0]$ и во втором случае вместо $x$ записьвая $-x$, придем к

$$
z^{(n)}(x)+\widetilde{N} z^{(n-1)}(x)=\lambda \widetilde{B} z(x)
$$


где $\widetilde{B}=\left(\begin{array}{ll}0 & 1 \\ 1 & 0\end{array}\right)$. Положим $u(x)=\Gamma^{-1} z(x)$. Тогда (8) переходит в

$$
L u(x)=\lambda u(x) .
$$

Займемся получением граничных условий (5) для $u(x)$. По теореме 1

$$
z_{1}^{(k-1)}\left(\frac{1}{2}\right)=0, \quad k=1, \ldots, n
$$

и, кроме того, очевидно, что $z_{1}^{(k-1)}(0)=(-1)^{k-1} z_{2}^{(k-1)}(0), k=1, \ldots, n$. В матричном виде эти соотношения запишутся так:

$$
\left(\begin{array}{cc}
1 & (-1)^{k} \\
0 & 0
\end{array}\right) z^{(k-1)}(0)+\left(\begin{array}{ll}
0 & 0 \\
1 & 0
\end{array}\right) z^{(k-1)}\left(\frac{1}{2}\right)=0, \quad k=1, \ldots, n .
$$

Выполнив здесь подстановку $z(x)=\Gamma u(x)$, придем к $(5)$.

Обратно, пусть $u(x)$ удовлетворяет $(9)$ и (5). Рассуждая в обратном порядке, получим, что $y(x)$, определяемая из $u(x)=\Gamma^{-1}(y(1 / 2+x), y(1 / 2-x))^{T}$, удовлетворяет $A^{-1} y(x)=\lambda y(x)$. Теорема доказана.

2. Резольвента оператора $L$.

1. Сначала займемся резольвентой $R_{0, \lambda}=\left(L_{0}-\lambda E\right)^{-1}$ оператора $L_{0}$ :

$$
L_{0} y=B y^{(n)}(x),
$$

где вектор-функция $y(x)$ удовлетворяет краевьм условиям

$$
U(y)=0
$$

и $U(y)=\left(U_{1}^{T}(y), \ldots, U_{n}^{T}(y)\right)^{T}$.

Положим $\lambda=\rho^{n}$, где $\arg \rho \in[0,2 \pi / n]$. Последний сектор разобьем на четыре сектора $S_{j}, j=1,2,3,4$, где $S_{j}=\left\{\rho \mid \arg \rho \in\left[\gamma_{j-1}, \gamma_{j}\right]\right\}, \gamma_{0}=0, \gamma_{1}=\pi / 2 n, \gamma_{2}=\pi / n$, $\gamma_{3}=3 \pi / 2 n, \gamma_{4}=2 \pi / n$, и для определенности будем рассматривать сектор $S_{1}$ (остальные рассматриваются аналогично). Обозначим через $\omega_{k}, k=1, \ldots, n,-$ корни $n$-й степени из 1 , занумерованные так, чтобы

$$
\operatorname{Re} \rho \omega_{1} \geqslant \operatorname{Re} \rho \omega_{2} \geqslant \cdots \geqslant \operatorname{Re} \rho \omega_{n / 2} \geqslant 0 \geqslant \operatorname{Re} \rho \omega_{n / 2+1} \geqslant \cdots \geqslant \operatorname{Re} \rho \omega_{n},
$$

и через $\omega_{k}, k=n+1, \ldots, 2 n,-$ корни $n$-й степени из -1 , для которых

$$
\operatorname{Re} \rho \omega_{n+1} \geqslant \cdots \geqslant \operatorname{Re} \rho \omega_{3 n / 2} \geqslant 0 \geqslant \operatorname{Re} \rho \omega_{3 n / 2+1} \geqslant \cdots \geqslant \operatorname{Re} \rho \omega_{2 n} .
$$

Рассмотрим функции

$$
\begin{aligned}
g_{1}(x, t, \rho)= & \frac{1}{n \rho^{n-1}}\left\{\varepsilon(x, t) \sum_{k=n / 2+1}^{n} \omega_{k} \exp \rho \omega_{k}(x-t)\right. \\
& \left.-\varepsilon(t, x) \sum_{k=1}^{n / 2} \omega_{k} \exp \rho \omega_{k}(x-t)\right\}, \\
g_{2}(x, t, \rho)= & \frac{1}{n \rho^{n-1}}\left\{\varepsilon(x, t) \sum_{k=3 n / 2+1}^{2 n} \omega_{k} \exp \rho \omega_{k}(x-t)\right. \\
& \left.-\varepsilon(t, x) \sum_{k=n+1}^{3 n / 2} \omega_{k} \exp \rho \omega_{k}(x-t)\right\},
\end{aligned}
$$


и через $g(x, t, \rho)$ обозначим матрицу-функцию

$$
g(x, t, \rho)=\left(\begin{array}{cc}
g_{1}(x, t, \rho) & 0 \\
0 & g_{2}(x, t, \rho)
\end{array}\right) .
$$

Лемма 1. Eсли

$$
y(x, \rho)=\int_{0}^{1 / 2} g(x, t, \rho) f(t) d t,
$$

то $y(x, \rho)$ удовлетворяет уравнению

$$
B y^{(n)}(x, \rho)-\lambda y(x, \rho)=f(x) .
$$

Это утверждение очевидно.

Обозначим

$$
\begin{aligned}
& \sigma_{k}(x, \rho)=\exp \rho \omega_{k}\left(x-\frac{1}{2}\right), \quad k=1, \ldots, \frac{n}{2}, n+1, \ldots, \frac{3 n}{2}, \\
& \sigma_{k}(x, \rho)=\exp \rho \omega_{k} x, \quad k=\frac{n}{2}+1, \ldots, n, \frac{3 n}{2}+1, \ldots, 2 n .
\end{aligned}
$$

Тогда система $\left\{\sigma_{k}(x, \rho)\right\}_{k=1}^{2 n}$ линейно независима и $\left|\sigma_{k}(x, \rho)\right| \leqslant 1$.

ЛЕмма 2. Общее решение уравнения (13) имеет вид

$$
y(x, \rho)=Y(x, \rho) c+\int_{0}^{1 / 2} g(x, t, \rho) f(t) d t
$$

əде $Y(x, \rho)=\left(Y_{1}(x, \rho), \ldots, Y_{n}(x, \rho)\right), Y_{k}(x, \rho)=\left(\begin{array}{cc}\sigma_{k}(x, \rho) & 0 \\ 0 & \sigma_{n+k}(x, \rho)\end{array}\right), c=\left(c_{1}, \ldots, c_{2 n}\right)^{T}$, и $c_{k}$ - произвольные постоянные.

Утверждение леммы следует из леммы 1 и того, что $Y_{k}(x, \rho), k=1, \ldots, n$, образуют фундаментальную систему решений однородного матричного уравнения

$$
B Y^{(n)}-\lambda Y=0
$$

ЛЕмма 3. Для резольвенты $R_{0, \lambda}$ имеет место формула

$$
R_{0, \lambda} f=\int_{0}^{1 / 2} G_{0}(x, t, \lambda) f(t) d t
$$

$2 \partial e$

$$
\begin{gathered}
G_{0}(x, t, \lambda)=g(x, t, \rho)+G_{1}(x, t, \rho) \\
G_{1}(x, t, \rho)=-Y(x, \rho) U^{-1}(Y) U(g(x, t, \rho)) \\
U(Y)=\left(\frac{1}{\rho^{j-1}} U_{j}\left(Y_{k}\right)\right)_{j, k=1}^{n}, \quad U(g)=\left(U_{1}^{T}(g), \frac{1}{\rho} U_{2}^{T}(g), \ldots, \frac{1}{\rho^{n-1}} U_{n}^{T}(g)\right)^{T}
\end{gathered}
$$

$u U_{j}$ применяется $\kappa g(x, t, \rho)$ по переменной $x$. 
ДокАЗАТЕЛЬСТво. Положим $y(x, \rho)=R_{0, \lambda} f(x)$. Тогда $y(x, \rho)$ удовлетворяет $(13)$ и условиям (12). По лемме 2 для $y(x, \rho)$ имеет место представление (14). Постоянный вектор $c$ находится из условий $(12)$, которые нам удобно записать в таком виде: $U_{j}(y) / \rho^{j-1}=0, j=1, \ldots, n$. Тогда

$$
U(Y) c+\int_{0}^{1 / 2} U(g(x, t, \rho)) f(t) d t=0 .
$$

Находя отсюда $c$ и подставляя в $(14)$, получим $(15),(16)$. Лемма доказана.

Лемма 4. Определитель $\Delta(\rho)$ матрищь $U(Y)$ имеет следующую асимптотику:

$$
\Delta(\rho)=a+b \exp \rho i+O\left(\exp \left(\frac{1}{2} \rho \omega_{3 n / 2+1}\right)\right),
$$

где а и

ДокАЗАТЕЛЬСТво. Обозначим через $\Delta_{1}(\rho)$ определитель, получающийся из $\Delta(\rho)$ заменой элементов, содержащих экспоненты, кроме $\exp \left(\frac{1}{2} \rho \omega_{n / 2+1}\right)$ и $\exp \left(-\frac{1}{2} \rho \omega_{n / 2}\right)$, на нуль. Тогда, так как все элементы $\Delta(\rho)$ ограничены по $\rho$, получим

$$
\Delta(\rho)=\Delta_{1}(\rho)+O\left(\exp \left(\frac{1}{2} \rho \omega_{3 n / 2+1}\right)\right) .
$$

В $\Delta_{1}(\rho)$ переставим столбцы так, чтобы элементы $i$-й строки снизу, стоящие в столбцах с номерами $n(i-1) / 2+k, k=1,2, \ldots, n / 2$, совпали при $i=1,2 \mathrm{c} \omega_{n(i-1) / 2+k}^{n-1}$, а при $i=3,4-\mathrm{c} \omega_{n(i-1) / 2+k}^{n-2}$. Полученньй определитель обозначим $\Delta_{2}(\rho)$. Ясно, что $\Delta_{1}(\rho)= \pm \Delta_{2}(\rho)$. В $\Delta_{2}(\rho)$ переставим строки так, чтобы в каждой из последних $n / 2$ строк первые $3 n / 2$ элементов равнялись нулю и в строках с номерами $n+1, \ldots, 3 n / 2$ каждьй из первых $n / 2-1$ элементов равнялся нулю. Полученньй определитель обозначим через $\Delta_{3}(\rho)$. Таким образом, $\Delta_{2}(\rho)= \pm \Delta_{3}(\rho)$. Далее, в определителе $\Delta_{3}(\rho)$ все элементы последних $n / 2$ строк, кроме элементов последних $n / 2$ столбцов, равны нулю, а минор $\gamma$, состоящий из элементов на пересечении последних $n / 2$ строк и последних $n / 2$ столбцов, есть определитель Вандермонда, образованньй из $\omega_{k}^{2}, k=3 n / 2+1, \ldots, 2 n$, и поэтому $\gamma \neq 0$. Поэтому по теореме Лапласа $\Delta_{3}(\rho)=\gamma \Delta_{4}(\rho)$, где $\Delta_{4}(\rho)$ есть определитель, состоящий из элементов, стоящих на пересечении первых $3 n / 2$ строк и первых $3 n / 2$ столбцов определителя $\Delta_{3}(\rho)$. Рассмотрим матрицу из последних $n / 2$ строк определителя $\Delta_{4}(\rho)$. Все элементы этих строк, кроме элементов, стоящих на пересечении со столбцами с номерами $n / 2, \ldots, n$, равны нулю. Поскольку $\omega_{n / 2}=-i$, то столбец с номером $n / 2$ этой матрицы есть

$$
\left(-i \exp \frac{\rho i}{2},(-i)^{3} \exp \frac{\rho i}{2}, \ldots,(-i)^{n-1} \exp \frac{\rho i}{2}\right)^{T}
$$

и столбцы с номерами $k=n / 2+1, \ldots, n$ образуют $\left(\omega, \omega_{k}^{3}, \ldots, \omega_{k}^{n-1}\right)^{T}$. Рассмотрим еще матрицу из первых $n$ строк определителя $\Delta_{4}(\rho)$. В ней столбцы с номерами $k=n / 2+2$, $\ldots, n$ состоят из нулей, столбцы с номерами $k=1, \ldots, n / 2, n+1, \ldots, 3 n / 2$ образуют $\left(1, \omega_{k}, \ldots, \omega_{k}^{n-1}\right)^{T}$. Так как $\omega_{n / 2+1}=i$, то столбец с номером $k=n / 2+1$ есть

$$
\left(\exp \frac{\rho i}{2}, i \exp \frac{\rho i}{2}, \ldots, i^{n-1} \exp \frac{\rho i}{2}\right)^{T} \text {. }
$$


Поэтому, разлагая определитель $\Delta_{4}(\rho)$ по минорам последних $n / 2$ строк, получим

$$
\Delta_{4}(\rho)=a_{1}+b_{1} \exp \rho i
$$

где $a_{1}, b_{1}$ отличны от нуля, так как представляют собой произведения ненулевых множителей на определители Вандермонда либо чисел $\omega_{k}$, либо $\omega_{k}^{2}$. Из (18) и (19) получаем (17). Лемма доказана.

Обозначим через $S_{1, \delta}$ область, получающуюся удалением из $S_{1}$ всех точек $\rho_{m}=$ $-i \ln (-a / b)+2 m \pi, m=0,1,2, \ldots$, являюшихся нулями функции $a+b \exp \rho i$, вместе с круговыми окрестностями $\gamma_{m}$ одного и того же достаточно малого радиуса $\delta$.

Лемма 5. В области $S_{1, \delta}$ при достаточно больиих $|\rho|$ для $G_{0}(x, t, \lambda)$ имеет место асимптотическая формула

$$
G_{0}(x, t, \lambda)=g(x, t, \rho)+\frac{1}{\rho^{n-1}} T(x, t, \rho),
$$

әде компоненты матрицы $T(x, t, \rho)$ являются линейными комбинациями функиий $\sigma_{k}(x, \rho) \sigma_{l}(1 / 2-t, \rho)$ с коэффициентами, являюшимися ограниченными функииямu $\rho$.

ДокАЗАТЕЛЬСТво. По лемме 4 при $\rho \in S_{1, \delta}$ имеет место при больших $|\rho|$ оценка $|\Delta(\rho)| \geqslant c>0$, где $c$ не зависит от $\rho$. Поэтому $U^{-1}(Y)$ представляет собой матрицу, элементы которой являются ограниченными функциями $\rho$. Далее, $\rho^{n-k} U_{k}(g(x, t, \rho))$, $k=1, \ldots, n$, представляют собой матрицы, элементы которых являются линейньпи комбинациями функций $\sigma_{j}(1 / 2-t, \rho)$ с постоянными коэффициентами. Утверждение леммы теперь есть простое следствие леммы 3.

СлЕДСТВИЕ. В области $S_{1, \delta}$ имеют место оценки

$$
\frac{\partial^{k}}{\partial x^{k}} G_{0 ; i j}(x, t, \lambda)=O\left(\frac{1}{\rho^{n-1-k}}\right), \quad k=0, \ldots, n-1, \quad i, j=1,2,
$$

где $G_{0 ; i j}(x, t, \lambda)$ - әлементы матрицы $G_{0}(x, t, \lambda)$.

2. Теперь изучим резольвенту $R_{\lambda}=(L-\lambda E)^{-1}$ оператора $L$.

Теорема 3. В области $S_{1, \delta}$ при больиих $|\rho|$ справедливо представление

$$
R_{\lambda}=R_{0, \lambda}-R_{0, \lambda} N(E+K)^{-1} D^{n-1} R_{0, \lambda},
$$

əде $K=D^{n-1} R_{0, \lambda} N, D=d / d x$.

ДоКАЗАТЕЛЬСТво. Пусть $y=R_{\lambda} f$. Тогда $B y^{(n)}-\lambda y=f-N y^{(n-1)}$. Отсюда

$$
y=R_{0, \lambda} f-R_{0, \lambda} N y^{(n-1)} \text {. }
$$

Дифференцируем (21) $n-1$ раз. Тогда

$$
y^{(n-1)}=D^{n-1} R_{0, \lambda} f-K y^{(n-1)} .
$$

Пусть $K(x, t, \rho)=\left(K_{i j}(x, t, \rho)\right)_{1}^{2}$ - ядро интегрального оператора $K$. Тогда имеем

$$
K_{i j}(x, t, \rho)=\sum_{k=1}^{2} \int_{0}^{1 / 2} \frac{\partial^{n-1}}{\partial x^{n-1}} G_{0 ; i, k}(x, \tau, \lambda) N_{k, j}(\tau, t) d \tau .
$$

Отсюда по лемме 5 и лемме $4[19$, с. 389$]$ получаем, что $K_{i j}(x, t, \rho)=o(1)$ при $|\rho| \rightarrow \infty$ равномерно по $x, t$. Поэтому оператор $E+K$ обратим и из $(21),(22)$ легко следует $(20)$. Теорема доказана. 


\section{3. Вспомогательные предложения.}

1. Обозначим через $\sigma(x, \rho, m)$ одну из функций $\sigma_{i}(x, \rho+2 m \pi), \sigma_{i}(1 / 2-x, \rho+2 m \pi)$, $i=1, \ldots, 2 n ; w(x, t, \rho, m)$ - одну из функций $\varepsilon(x, t) \exp (\rho+2 m \pi) \omega(x-t)$, когда $\omega-$ одно из чисел $\omega_{k}, \omega_{n+k}$ при $k=n / 2+1, \ldots, n ; \varepsilon(t, x) \exp (\rho+2 m \pi) \omega(x-t)$, ког да $\omega-$ одно из $\omega_{k}, \omega_{n+k}$ при $k=1, \ldots, n / 2$. Имеет место очевидная формула

$$
\sigma(x, \rho, m)=\sigma(x, \rho, 0) \sigma(x, 0, m) .
$$

Операторы

$$
P_{m} g(x)=\int_{0}^{1 / 2} p(x, t, \rho, m) g(t) d t
$$

будем назьвать әлементарными. Здесь $p(x, t, \rho, m)=\sigma(x, \rho, m) \sigma(t, \rho, m)$ или $p(x, t, \rho$, $m)=w(x, t, \rho, m)$.

ЛЕмма 6. Если ядра всех операторов $P_{m}$ отличаются лишь параметром $m u$ $\rho$ принадлежит ограниченной области, то

$$
\sum_{m=1}^{\infty}\left\|P_{m} g\right\|^{2} \leqslant C\|g\|^{2}
$$

әде С не зависит от $\rho$ инормы берутся в $L_{2}[0,1 / 2]$.

ДокАЗАТЕЛЬСтво. Пусть сначала $p(x, t, \rho, m)=\sigma(x, \rho, m) \sigma(t, \rho, m)$. Тогда $P_{m} g=$ $\sigma(x, \rho, m) b_{m}(g, \rho)$, где

$$
b_{m}(g, \rho)=\int_{0}^{1 / 2} g(t) \sigma(t, \rho, m) d t .
$$

Так как в силу (23)

$$
b_{m}(g, \rho)=\int_{0}^{1 / 2} g_{1}(t, \rho) \sigma(t, 0, m) d t,
$$

где $g_{1}(t, \rho)=g(t) \sigma(t, \rho, 0)$, то ряд $\sum_{m=1}^{\infty}\left|b_{m}(g, \rho)\right|^{2}$ по лемме $3.2[9$, c. 213] сходится и его сумма ограничена по $\rho$. Поскольку $|\sigma(x, \rho, m)| \leqslant 1$, то $\left\|P_{m} g\right\| \leqslant\left|b_{m}(g, \rho)\right|$, и $(24)$ в этом случае установлено.

Пусть теперь $p(x, t, \rho, m)=w(x, t, \rho, m)$ и для определенности пусть $w(x, t, \rho, m)=$ $\varepsilon(x, t) \exp (\rho+2 m \pi) \omega(x-t)$. Тогда

$$
P_{m} g=\int_{0}^{x} g_{2}(x, t, \rho) \exp 2 m \pi \omega t d t
$$

где $g_{2}(x, t, \rho)=g(x-t) \exp \rho \omega t$. Фиксируем $x$. Тогда опять по лемме 3.2 [9]

$$
\sum_{m=1}^{\infty}\left|P_{m} g\right|^{2} \leqslant C \int_{0}^{x}|g(x-t)|^{2} d t \leqslant C\|g\|^{2},
$$

где $C$ не зависит от $x$ и $\rho$. Интегрируя это неравенство по $x$ от 0 до $1 / 2$, придем к (24). Лемма доказана.

2. Пусть $\lambda=\rho^{n}, \rho \in S_{1, \delta}, \rho=\rho_{1}+2 m \pi$ и $\rho_{1}$ принадлежит ограниченной области комплексной плоскости. 
Лемма 7. Каждая компонента вектор-функиий $\rho^{n-1-j} D^{j} R_{0, \lambda} f, j=0, \ldots, n-1$, есть конечная сумма с ограниченными по $\rho_{1}$ и $m$ коэффициентами элементарных операторов при всевозможсных вариантах ядер $p\left(x, t, \rho_{1}, m\right)$, причем если $p\left(x, t, \rho_{1}\right.$, $m)=w\left(x, t, \rho_{1}, m\right)$, то коэффициенты при $P_{m} g$ являются постоянными числами, не зависящ, ими от $\rho_{1} u m ; g(x)$ - одна из компонент вектора $f(x)=\left(f_{1}(x), f_{2}(x)\right)^{T}$.

ДокАЗАТЕЛЬСтво. По лемме 5 имеем

$$
D^{j} R_{0, \lambda} f=\int_{0}^{1 / 2} g_{x^{j}}^{(j)}(x, t, \rho) f(t) d t+\rho^{1-n} \int_{0}^{1 / 2} T_{x^{j}}^{(j)}(x, t, \rho) f(t) d t .
$$

Далее,

$$
\rho^{n-1-j} \int_{0}^{1 / 2} g_{x^{j}}^{(j)}(x, t, \rho) f(t) d t
$$

есть вектор-функция, компоненты которой есть линейные комбинации с постоянными коэффициентами операторов $P_{m} g$, когда $p\left(x, t, \rho_{1}, m\right)=w\left(x, t, \rho_{1}, m\right)$;

$$
\rho^{-j} \int_{0}^{1 / 2} T_{x^{j}}^{(j)}(x, t, \rho) f(t) d t
$$

есть вектор-функция, компоненты которой есть линейные комбинации операторов $P_{m} g$, когда $p\left(x, t, \rho_{1}, m\right)=\sigma\left(x, \rho_{1}, m\right) \sigma\left(t, \rho_{1}, m\right)$ с коэффициентами, являющимися ограниченньпми функциями по $m$ и $\rho_{1}$. Лемма доказана.

Пусть $\Gamma_{m}$ - образ в $\lambda$-плоскости окружности $\gamma_{m}$ из определения $S_{1, \delta}$ и без ограничения общности будем считать, что $\gamma_{m}$ находятся целиком в $S_{1}$ (в противном случае так же, как и в [20, с. 55], переходим к смещенному сектору). Тогда если $\rho \in \gamma_{m}$, то $\rho_{1}=\rho-2 m \pi$ принадлежит фиксированной окружности радиуса $\delta$. Пусть $I-$ любой конечньй набор достаточно больших номеров $m$.

Лемма 8. Имеет место оиенка

$$
\left\|\sum_{m \in I} \int_{\Gamma_{m}} R_{0, \lambda} d \lambda\right\| \leqslant C
$$

где $C$ не зависит от $I$ и норма берется в пространстве операторов в $L_{2}^{2}[0,1 / 2]$.

ДокАЗАТЕЛЬСТво. Имеем

$$
\sum_{m \in I} \int_{\Gamma_{m}} R_{0, \lambda} f d \lambda=\int_{\gamma} \Phi\left(f, \rho_{1}\right) d \rho_{1}
$$

где

$$
\Phi\left(f, \rho_{1}\right)=\left.n \sum_{m \in I} R_{0, \lambda} f\right|_{\lambda=\left(\rho_{1}+2 m \pi\right)^{n}}\left(\rho_{1}+2 m \pi\right)^{n-1} .
$$

По лемме 7 каждую компоненту вектор-функции $\Phi\left(f, \rho_{1}\right)$ представим в виде конечной суммы операторов

$$
\Sigma=\sum_{m \in I} \alpha\left(\rho_{1}, m\right) P_{m} g
$$


где $\alpha\left(\rho_{1}, m\right)$ - ограниченные функции по $\rho_{1}$ и $m$; ядра $p\left(x, t, \rho_{1}, m\right)$ состоят из одних и тех же функций, отличающихся лишш параметром $m$. Если $p\left(x, t, \rho_{1}, m\right)=w\left(x, t, \rho_{1}, m\right)$, то $\alpha\left(\rho_{1}, m\right)$ - константы, не зависящие от $\rho_{1}$ и $m$, и поэтому в этом случае $\int_{\gamma} \Sigma d \rho_{1}=0$. Поэтому рассмотрим лишь суммы $(25)$, когда $p\left(x, t, \rho_{1}, m\right)=\sigma\left(x, \rho_{1}, m\right) \sigma\left(t, \rho_{1}, m\right)$. В этом случае

$$
\Sigma=\sum_{m \in I} \alpha\left(\rho_{1}, m\right) \sigma_{m}\left(x, \rho_{1}, m\right) b_{m}\left(g, \rho_{1}\right),
$$

и покажем, что по норме $L_{2}[0,1 / 2]$

$$
\|\Sigma\| \leqslant C\|g\|
$$

причем константа $C$ не зависит от $I$ и $\rho_{1}$. Пусть $u(x) \in L_{2}[0,1 / 2]$. Тогда

$$
(\Sigma, u)=\sum_{m \in I} \alpha\left(\rho_{1}, m\right) b_{m}\left(u, \rho_{1}\right) b_{m}\left(g, \rho_{1}\right)
$$

Поэтому

$$
\begin{aligned}
|(\Sigma, u)| & \leqslant C \sum_{m=1}^{\infty}\left|b_{m}\left(u, \rho_{1}\right)\right|\left|b_{m}\left(g, \rho_{1}\right)\right| \\
& \leqslant C\left(\sum_{m=1}^{\infty}\left|b_{m}\left(u, \rho_{1}\right)\right|^{2}\right)^{1 / 2}\left(\sum_{m=1}^{\infty}\left|b_{m}\left(g, \rho_{1}\right)\right|^{2}\right)^{1 / 2}<\infty .
\end{aligned}
$$

Значит, по теореме Банаха-Штейнгауза функционалы $(\Sigma, u)$ имеют нормы $\|\Sigma\|$, ограниченные константой, не зависящей от $I$ и $\rho_{1}$. Теперь, применяя опять теорему Банаха-Штейнгауза к операторам $\Sigma$, получим оценку (26). Лемма доказана.

ЛЕмма 9. Имеет место оиенка

$$
\left\|\sum_{m \in I} \int_{\Gamma_{m}} R_{0, \lambda} N(E+K)^{-1} D^{n-1} R_{0, \lambda} d \lambda\right\| \leqslant C
$$

әде $C$ не зависит от $I$.

ДокАЗАТЕЛЬСТво. Как и при доказательстве предыдущей леммы, имеем

$$
\sum_{m \in I} \int_{\Gamma_{m}} R_{0, \lambda} N(E+K)^{-1} D^{n-1} R_{0, \lambda} d \lambda=\int_{\gamma} \Phi\left(f, \rho_{1}\right) d \rho_{1}
$$

где теперь

$$
\Phi\left(f, \rho_{1}\right)=\left.n \sum_{m \in I} R_{0, \lambda} N(E+K)^{-1} D^{n-1} R_{0, \lambda} f\right|_{\lambda=\left(\rho_{1}+2 m \pi\right)^{n}}=\left(\rho_{1}+2 m \pi\right)^{n-1}
$$

Каждую компоненту вектор-функции $\Phi\left(f, \rho_{1}\right)$ представим в виде суммы операторов вида

$$
\Sigma=\sum_{m \in I} \alpha\left(\rho_{1}, m\right) P_{m} M_{m} P_{m} g
$$


где $M_{m}$ - одна из компонент интегрального оператора $N(E+K)^{-1}$ при $\lambda=\left(\rho_{1}+2 m \pi\right)^{n}$ и ядра операторов $P_{m}$, стоящих перед $M_{m}$ в $P_{m} M_{m} P_{m}$, отличаются лиш параметром $m$ и таким же свойством обладают ядра операторов $P_{m}$ после $M_{m}$ в $P_{m} M_{m} P_{m}$. Пусть $u(x) \in L_{2}[0,1 / 2]$. Тогда

$$
(\Sigma, u)=\sum_{m \in I} \alpha\left(\rho_{1}, m\right)\left(M_{m} P_{m} g, P_{m}^{*} u\right)
$$

Так как ядра операторов $M_{m}$ ограничены по $m$ и $\rho_{1}$, отсюда получаем оценку

$$
|(\Sigma, u)| \leqslant C \sum_{m \in I}\left\|P_{m} g\right\|\left\|P_{m}^{*} u\right\| .
$$

Аналогично лемме 6 можно показать, что

$$
\sum_{m=1}^{\infty}\left\|P_{m}^{*} u\right\|^{2} \leqslant c\|u\|^{2}
$$

Поэтому из (27) в силу леммы 6 следует оценка $|(\Sigma, u)| \leqslant C\|g\|\|u\|$. Значит, $\|\Sigma\| \leqslant C$ и лемма доказана.

Из теоремы 3 и лемм 8,9 получаем

Теорема 4. Имеет место оиенка

$$
\left\|\sum_{m \in I} \int_{\Gamma_{m}} R_{\lambda} d \lambda\right\| \leqslant C
$$

равномерная по $I$.

4. Базисы Рисса из собственных и присоединенных функций. Теперь приступаем к установлению базисности по Риссу системы СП $\Phi$ оператора $A$.

Лемма 10. Все достаточно большие по модулю собственные значения оператора $L$ однократны.

ДокАЗАТЕЛЬСТво. По лемме 4 такие собственные значения оператора $L_{0}$, образы которых в $\rho$-плоскости попадают в $S_{1}$, однократны. Далее, воспользуемся теоремой 3 . Так как компоненты ядра оператора $R_{0, \lambda} N$ суть $o\left(\rho^{1-n}\right)$ при $|\rho| \rightarrow \infty$ и компоненты ядра $(E+K)^{-1} D^{n-1} R_{0, \lambda}$ суть $O(1)$, то в $S_{1, \delta}$ компоненты ядра $R_{\lambda}-R_{0, \lambda}$ суть $o\left(\rho^{1-n}\right)$ при $|\rho| \rightarrow \infty$. Но известно, что если

$$
R_{\lambda} f=\int_{0}^{1 / 2} G(x, t, \lambda) f(t) d t
$$

то $\operatorname{tr} A_{m}$, где

$$
A_{m}=-\frac{1}{2 \pi i} \int_{\Gamma_{m}} \int_{0}^{1 / 2} G(x, x, \lambda) d x d \lambda
$$


и $\operatorname{tr} A_{m}$ - след матрицы $A_{m}$ - равен кратности собственного значения $\lambda_{m}$, а так как при больших $m \operatorname{tr} A_{m}^{0}$, где

$$
A_{m}^{0}=-\frac{1}{2 \pi i} \int_{\Gamma_{m}} \int_{0}^{1 / 2} G_{0}(x, x, \lambda) d x d \lambda,
$$

равен 1, то и $\operatorname{tr} A_{m}=1$. Аналогичное рассуждение справедливо и для тех $\lambda_{m}$, образы которых в $\rho$-плоскости не попадают в $S_{1}$. Лемма доказана.

Положим

$$
E\left(\lambda_{m}\right)=-\frac{1}{2 \pi i} \int_{\Gamma_{m}} R_{\lambda} d \lambda
$$

где теперь $\lambda_{m}, m=1,2 \ldots,-$ все собственные значения оператора $L$ и $\Gamma_{m}$ - замкнутьй контур в $\lambda$-плоскости, содержащий лиш одно собственное значение $\lambda_{m}$.

ЛЕмма 11. Если $f(x) \in L_{2}^{2}[0,1 / 2] u E\left(\lambda_{m}\right) f=0, m=1,2, \ldots$, mo $f(x)=0$ nочтu всюду.

ДокАЗАТЕльство. Если $E\left(\lambda_{m}\right) f=0, m=1,2, \ldots$, то $R_{\lambda} f$ - целая по $\lambda$, а в силу теоремы 3 и следствия леммы 5 отсюда по теореме Лиувилля $R_{\lambda} f \equiv 0$. Отсюда $f(x)=0$ почти всюду.

ЛЕмма 12. Если система вектор-функиий $\left\{\left(\varphi_{k} x\right)\right\}_{1}^{\infty}$, әде

$$
\varphi_{k}(x)=\left(\varphi_{1, k}(x), \varphi_{2, k}(x)\right)^{T}, \quad k=1,2, \ldots,
$$

образует базис Рисса в $L_{2}^{2}[0,1 / 2]$, то система $\left\{g_{k}(x)\right\}_{1}^{\infty}$, где $g_{k}(x)=\varphi_{2, k}(1 / 2-x)$ при $x \in[0,1 / 2]$ u $g_{k}(x)=\varphi_{1, k}(x-1 / 2)$ при $x \in[1 / 2,1]$, образует базис Рисса в $L_{2}[0,1]$.

ДокАЗАТЕЛЬСтво. Покажем сначала, что система $\left\{r_{k}(x)\right\}_{1}^{\infty}$, где $r_{k}(x)=\varphi_{1, k}(x)$ при $x \in[0,1 / 2]$ и $r_{k}(x)=\varphi_{2, k}(-x)$ при $x \in[-1 / 2,0]$, образует базис Рисса в $L_{2}[-1 / 2$, $1 / 2]$. В самом деле, пусть $f(x) \in L_{2}[-1 / 2,1 / 2]$. Образуем $F(x)=\left(F_{1}(x), F_{2}(x)\right)^{T}$, где $F_{1}(x)=f(x), F_{2}(x)=f(-x)$ и $x \in[0,1 / 2]$. Пусть система вектор-функций $\left\{\psi_{k}(x)\right\}_{1}^{\infty}$, где $\psi_{k}(x)=\left(\psi_{1, k}(x), \psi_{2, k}(x)\right)^{T}$, биортогональна системе $\left\{\varphi_{k}(x)\right\}_{1}^{\infty}$. Тогда имеет место разложение

$$
F(x)=\sum_{k=1}^{\infty}\left(F, \psi_{k}\right)_{2} \varphi_{k}(x)
$$

где $\left(F, \psi_{k}\right)_{2}$ - скалярное произведение в $L_{2}^{2}[0,1 / 2]$.

Положим $u_{k}(x)=\psi_{1, k}(x)$ при $x \in[0,1 / 2]$ и $u_{k}(x)=\psi_{2, k}(-x)$ при $x \in[-1 / 2,0]$. Тогда $\left(F, \psi_{k}\right)_{2}=\left(f, u_{k}\right)$, где $\left(f, u_{k}\right)$ - скалярное произведение в $L_{2}[-1 / 2,1 / 2]$. Поэтому (28) переходит в

$$
f(x)=\sum_{k=1}^{\infty}\left(f, u_{k}\right) r_{k}(x) .
$$

Далее, $\left(r_{k}, u_{s}\right)=\left(\varphi_{k}, \psi_{s}\right)_{2}=\delta_{k, s}$. Поэтому (29) представляет собой разложение Фурье функции $f(x)$ по системе $\left\{r_{k}(x)\right\}_{1}^{\infty}$, для которой $\left\{u_{k}(x)\right\}_{1}^{\infty}$ является биортогональной. Так как

$$
\sum_{k=1}^{\infty}\left|\left(f, u_{k}\right)\right|^{2}=\sum_{k=1}^{\infty}\left|\left(F, \psi_{k}\right)_{2}\right|^{2}<\infty, \quad \sum_{k=1}^{\infty}\left|\left(f, r_{k}\right)\right|^{2}=\sum_{k=1}^{\infty}\left|\left(F, \varphi_{k}\right)_{2}\right|^{2}<\infty,
$$

то $\left\{r_{k}(x)\right\}_{1}^{\infty}$ образует базис Рисса в $L_{2}[-1 / 2,1 / 2]$. А тогда система $\left\{g_{k}(x)\right\}_{1}^{\infty}$ образует базис Рисса в $L_{2}[0,1]$. Лемма доказана. 
Теорема 5. Система СПФ оператора А образует базис Рисса в $L_{2}[0,1]$.

ДокАЗАтЕльСтво. Пусть $E^{*}\left(\lambda_{k}\right)$ - оператор, сопряженный к $E\left(\lambda_{k}\right)$. Покажем, что ряд

$$
\sum_{k=1}^{\infty} E^{*}\left(\lambda_{k}\right) f
$$

безусловно сходится к $f(x)$ в $L_{2}^{2}[0,1 / 2]$. Пусть уже в (30) система $\left\{\lambda_{k}\right\}_{1}^{\infty}$ записана в каком-нибудь наперед заданном порядке. По лемме 11 система СП $\Phi\left\{\psi_{k}(x)\right\}_{1}^{\infty}$ оператоpa $L^{*}$ полна в $L_{2}^{2}[0,1 / 2]$. Зададим $\varepsilon>0$. Тогда существуют числа $\alpha_{k}, k=1, \ldots, m$, и номер $m$ такие, что

$$
\left\|f-\sum_{k=1}^{m} \alpha_{k} \psi_{k}\right\| \leqslant \varepsilon .
$$

Пусть

$$
S_{q}=\sum_{k=1}^{q} E^{*}\left(\lambda_{k}\right) .
$$

Тогда при $q>m$

$$
\begin{aligned}
\left\|f-S_{q} f\right\| & \leqslant\left\|f-\sum_{k=1}^{m} \alpha_{k} \psi_{k}\right\|+\left\|\sum_{k=1}^{m} \alpha_{k} \psi_{k}-S_{q}\left(\sum_{k=1}^{m} \alpha_{k} \psi_{k}\right)\right\|+\left\|S_{q}\left(f-\sum_{k=1}^{m} \alpha_{k} \psi_{k}\right)\right\| \\
& \leqslant \varepsilon+\left\|S_{q}\right\| \varepsilon \leqslant(1+c) \varepsilon
\end{aligned}
$$

так как по теореме $4\left\|S_{q}\right\|$ ограничена по $q$. Отсюда по лемме 10 следует, что система $\left\{\psi_{k}(x)\right\}_{1}^{\infty}$ образует перестановочньй базис, а система $\left\{\psi_{k}(x) /\left\|\psi_{k}\right\|\right\}_{1}^{\infty}$ - базис Рисса. Следовательно, биортогональная к этой последней системе, которую обозначим через $\left\{\varphi_{k}(x)\right\}_{1}^{\infty}$ и которая состоит из СП $\Phi$ оператора $L,-$ тоже базис Рисса. По лемме 12 система $\left\{g_{k}(x)\right\}_{1}^{\infty}$, где $g_{k}(x)=\left(\Gamma \varphi_{k}(1 / 2-x)\right)_{2}$ при $x \in[0,1 / 2]$ и $g_{k}(x)=\left(\Gamma \varphi_{k}(x-1 / 2)\right)_{1}$ при $x \in[1 / 2,1]$, образует базис Рисса в $L_{2}[0,1]$ и в то же время по теореме 2 она является системой СП $\Phi$ оператора $A$. Теорема доказана.

Авторы выражают благодарность А. А. Шкаликову за ценные замечания.

\section{СПИСОК ЦИТИРОВАННОЙ ЛИТЕРАТУРЫ}

[1] Хромов А. П. Об обращении интегральных операторов с ядрами, разрывными на диагоналях // Матем. заметки. 1998. Т. 64. №6. С. 932-942.

[2] Хромов А. П. Теорема равносходимости для интегрального оператора с переменным верхним пределом интегрирования // Сб. статей, посвященньй 70-летию П. Л. Ульянова. М., 1999. C. $255-266$.

[3] Корнев В.В., Хромов А. П. О равносходимости разложений по собственньм функциям интегральных операторов с ядрами, допускающими разрывы производных на диагоналях // Матем. сб. 2001. Т. 192. № 10. С. 33-50.

[4] Михайлов В.П. О базисах Рисса в $L_{2}[0,1]$ // Докл. АН СССР. 1962. Т. 144. №5. C. $981-984$.

[5] Кесельман Г. М. О безусловной сходимости разложений по собственньм функциям некоторых дифференциальных операторов // Изв. вузов. Матем. 1964. №2 2. С. 82-93.

[6] Данфорд Н., Шварц Дж. Т. Линейные операторы. Т. 3. М.: Мир, 1974. 
[7] Шкаликов А.А. О базисности собственных функций обыкновенного дифференциального оператора // УМН. 1979. Т. 34. № 5. С. 235-236.

[8] Шкаликов А.А. О базисности собственных функций обыкновенных дифференциальных операторов с интегральными краевыми условиями // Вестн. МГУ. Сер. матем., мех. 1982. №6. C. $12-21$.

[9] Шкаликов А. А. Краевые задачи для обыкновенных дифференциальных уравнений с параметром в граничных условиях // Тр. семинара им. И. Г. Петровского. 1983. Т. 9. С. 190-229.

[10] Гомилко А. М., Радзиевский Г. В. Базисные свойства собственных функций регулярной краевой задачи для векторного функционально-дифференциального уравнения // Дифференц. уравнения. 1991. Т. 27. № 3. С. 384-396.

[11] Радзиевский Г. В.Краевые задачи и связанные с ними модули непрерывности // Функцион. анализ и его прилож. 1995. Т. 29. №3. С. 87-90.

[12] Баскаков А. Г., Кацаран Т. К. Спектральный анализ интегро-дифференциальных операторов с нелокальными краевыми условиями // Дифференц. уравнения. 1988. Т. 24. № 8 . C. $1424-1433$.

[13] Ильин В.А. Необходимые и достаточные условия базисности в $L_{p}$ и равносходимости с тригонометрическим рядом спектральных разложений и разложений по системам экспонент // Докл. АН СССР. 1983. Т. 273. № 4. С. 789-793.

[14] Ильин В. А. Необходимые и достаточные условия базисности и равносходимости с тригонометрическим рядом спектральных разложений I // Дифференц. уравнения. 1980. Т. 16. № 5 . C. 771-794.

[15] Ильин В. А. Необходимые и достаточные условия базисности и равносходимости с тригонометрическим рядом спектральных разложений II // Дифференц. уравнения. 1980. Т. 16. №6. C. $980-1009$.

[16] Пальцев Б. В. Разложения по собственным функциям интегральных операторов свертки на конечном интервале с ядрами, преобразования Фурье которых рациональны. "Слабо несамосопряженные регулярные ядра" // Изв. АН СССР. Сер. матем. 1972. Т. 36. № 3. С. 591-634.

[17] Курдюмов В. П. Необходимые и достаточные условия оптимальности для дифференциально-операторного уравнения. Деп. ВИНИТИ 02.07.85 № 4781. Саратов: Сарат. ун-т, 1985.

[18] Курдюмов В. П. О базисах Рисса из собственных и присоединенных функций некоторых интегральных операторов // Дифференц. уравнения. 2002. Т. 38. № 4. С. 555-564.

[19] Хромов А. П. Теоремы равносходимости для интегро-дифференциальных и интегральных операторов // Матем. сб. 1981. Т. 114(156). №3. С. 378-405.

[20] Наймарк М. А. Линейные дифференциальные операторы. М.: Наука, 1969.

Саратовский государственный университет им. Н. Г. Чернышевского

Поступило

E-mail: KhromovAPQinfo.sgu.ru

25.11 .2002

Исправленный вариант

03.11 .2003 